\title{
Potential Role of microRNA-183 as a Tumor Suppressor in Hepatocellular Carcinoma
}

\author{
Wei Bian ${ }^{\mathrm{a}}$ Hongfei Zhang ${ }^{\mathrm{b}}$ Miao Tang ${ }^{\mathrm{b}}$ Shaojun Zhang Lichao Wang $^{\mathrm{b}}$ \\ Longlong Liu ${ }^{b}$ Wenyao Wang \\ aDepartment of Hepatobiliary Surgery, The Second Hospital of Hebei Medical University, Shijiazhuang, \\ bDepartment of General Surgery of East District, The Second Hospital of Hebei Medical University, \\ Shijiazhuang, China
}

\author{
Key Words \\ microRNA-183 • Tumor Suppressor • Hepatocellular Carcinoma
}

\begin{abstract}
Background/Aims: Disseminated tumors, known as metastases, are responsible for ninetypercent of mortality due to cancer. Epithelial to mesenchymal transition, a phenomenon required for morphological conversion of non-motile discoid shaped epithelial cells to highly motile spindle-shaped mesenchymal cells, is thought to be a pre-requisite for metastatic progression. Metastasis-associated 1 (MTA1) protein is a prime inducer of EMT and metastatic progression in all solid tumors including hepatocellular carcinoma (HCC). However, the molecular mechanisms that regulate the expression and function of MTA1 in HCC have not been elucidated. Methods: In silico prediction algorithms were used to find microRNAs (miRNAs) that may target MTA1. We examined the relationship between the expression of MTA1 and miR-183 using quantitative real time PCR. We also determined the levels of the MTA1 protein using immunohistochemistry. Reporter assays, in the presence and absence of the miR-183 mimic, were used to confirm MTA1 as a bona fide target of miR183. The effect of miR-183 on HCC pathogenesis was determined using a combination of in vitro migration and invasion assay, together with in vivo xenograft experiments. The correlation between miR183 and MTA 1 expression was also studied in samples from HCC patients, and in The Cancer Genome Atlas dataset. Results: Analysis of the sequence database revealed that MTA1 is a putative target of miR-183. MTA1 protein and RNA expression showed opposite trends to miR183 expression in breast, renal, prostate, and testicular tissue samples from cancer patients, and in the metastatic HCC cell line HepG2. An inverse correlation was also observed between MTA 1 (high) and miR-183 (low) expression within samples from HHC patients and in the TCGA dataset. Reporter assays in HepG2 cells showed that miR-183 could inhibit translation of a reporter harboring the wild-type, but not the mutant miR-183 3'-untranslated region (UTR). In addition, miR-183 significantly inhibited in vitro migration and invasion in HepG2 cells, and in
\end{abstract}


vivo hepatic metastasis. Conclusion: Our results reveal a novel post-transcriptional regulatory mechanism for MTA1 expression via miR-183, which is suppressed during HCC pathogenesis.

(c) 2018 The Author(s)

Published by S. Karger AG, Basel

\section{Introduction}

The incidence of hepatocellular carcinoma (HCC) is increasing. It has become a frequently encountered form of solid cancer [1-3]. Advances made over the last two decades are aiding early diagnosis, therapy, and surgical management; unfortunately, however, all efforts have so far failed to contain HCC - due to its high propensity to metastasize to secondary organs and its resistance to chemotherapy $[4,5]$. Therefore, it is important to identify prognostic and diagnostic molecular markers in HCC.

The metastasis-associated (MTA) gene family -has three members, namely MTA1, MTA2 and MTA3 [6]. MTA1 is an intrinsic component of the nucleosome remodeling and histone deacetylation complex and can function as a transcription factor $[7,8]$. Importantly, MTA1 is a central regulator of epithelial-mesenchymal transition (EMT) [7]. It has been shown that MTA1 overexpression leads to a poor outcome in patients with malignant tumors $[9,10]$. However, what precisely regulates MTA1 expression is not currently known; although it has been found that the estrogen receptor alpha (ER $\alpha$ ) inhibits MTA1 transcription and, as a result, retards invasion and proliferation during HCC pathogenesis [11].

MicroRNAs, a group of endogenous, small noncoding RNAs, can regulate numerous normal cellular pathways as well as aberrant processes during tumor pathogenesis. MicroRNAs can inhibit target gene function by either promoting degradation of the mRNA or by suppressing its translation, with the degree of complementarity between the miRNA and target mRNA sequences directing which of the two modes of inhibition will take effect [12]. MiRNAs possess both tumor suppressive and oncogenic functions [13]; a great deal of work is needed to understand why and how they influence tumor progression. The objective of this study was to determine whether miRNAs regulate MTA1 expression in HCC.

\section{Materials and Methods}

\section{Patients and tissue processing}

The study design was approved by the Institutional Review Board of the Second Hospital of Hebei Medical University, China. Either paraffin-embedded or fresh snap frozen samples with a pathological diagnosis of breast, renal, prostate, and testicular tissue specimens ( $n=10$ per cancer type) were obtained from Chinese cancer patients at the Second Hospital of Hebei Medical University between 2014 and 2015. We chose patients for inclusion based on confirmed diagnosis, no prior adjuvant or chemotherapy, availability of follow-up, and clinical data. Freshly harvested tissue specimens were equilibrated with RNAlater (Life Technologies, Shanghai, China) before being snap frozen. All samples were stored in liquid nitrogen tanks for long-term storage.

\section{Cell culture}

HepG2 cell line was obtained from ATCC (Beijing Zhongyuan Ltd, Beijing, China). Media for HepG2 was FBS (10\%, Lonza, Germany) and penicillin/streptomycin (ThermoFisher Scientific, Shanghai, China) supplemented with Dulbecco's Modified Eagle Medium (DMEM) (ThermoFisher Scientific).

Isolation of RNA and miRNA extraction and quantitative real time polymerase chain reaction (qRT-PCR)

RNA was isolated using Trizol (ThermoFisher Scientific) according to the manufacturer's protocol. The following TaqMan Gene Expression probes (ThermoFisher Scientific) were used for qRT-PCR: MTA1 (TaqMan Assay ID: Hs00950776_m1) and TBP (TaqMan Assay ID: Hs00427620_m1). Data analysis was performed using the $-\triangle \triangle \mathrm{Ct}$ method. Relative changes were normalized to TBP expression. The mirVana miRNA isolation kit (ThermoFisher Scientific) was used to isolate miRNA. The following TaqMan miRNA 


\section{Cellular Physiology Cell Physiol Biochem 2018;51:2065-2072 and Biochemistry \begin{tabular}{l|l} 
DOI: 10.1159/000495825 & $\begin{array}{l}\text { O } 2018 \text { The Author(s). Published by S. Karger AG, Basel } \\
\text { mwww.karger.com/cpb }\end{array}$
\end{tabular} \\ Bian et al.: microRNA-183 Functions in HCC}

assay probes were used for qRT-PCR: miR-183 (TaqMan Assay ID: 002270) and RNU6B (TaqMan Assay ID: 001093). The latter was used for data normalization.

\section{Immunohistochemistry}

Tissue specimens from representative patients ( $\mathrm{n}=3$ in each case) with breast, renal, prostate and testicular carcinoma with high and low miR-183 levels were stained for MTA1 expression (\#ab-71153, Abcam, Cambridge, UK). A score of 'low' or 'high' was assigned to each specimen by a pathologist blinded to the identity of the specimens.

\section{Gene construction}

The MTA1 3' UTR was cloned into the XhoI and ApaI sites in the Rr-luc-6XCXCR4 Renilla luciferase vector (Addgene, Beijing Zhongyuan Ltd. Beijing, China). Site-directed mutagenesis was used to delete the 321-327 region, corresponding to the hsa-miR-183 binding site, in the reporter plasmid. For transfection control, a firefly luciferase gene, not under the control of MTA1 3'UTR, was used.

\section{Transfection and luciferase assays}

HepG2 cells were transiently transfected with Rr-luc-6XCXCR4 Renilla luciferase vector using Lipofectamine 2000 (ThermoFisher Scientific) according to the manufacturer's protocol. For the mimics, the cells were also co-transfected with either C-X-C chemokine receptor type 4 (CXCR-4) or the miR-183 mimic (ThermoFisher Scientific). Luciferase assays were performed 96 hours after transfection, using the Dual-luciferase assay kit (Promega, Madison, Wisconsin, USA). Results shown are from three biological replicates, each performed with three technical replicates. Data are shown as relative fluorescence units $(\mathrm{RFU}) \pm$ standard deviation (SD).

\section{Cell proliferation assay}

The MTT assay kit (Sigma-Aldrich, Shanghai, China) was used to determine cell proliferation quantitatively. Results, shown as relative optical density (OD) (mean \pm standard deviation), are from three biological replicates, each performed with three technical replicates.

Trans-well migration and invasion assays

Migration and invasion assays were performed using Culture kits (R\&D Systems, Shanghai, China). Results shown are from three biological replicates, each carried out with three technical replicates.

\section{Xenograft assay}

The Institutional Animal Care and Use Committee of the Second Hospital of Hebei Medical University approved all animal studies performed and reported in this manuscript. FF-HepG2 cells $\left(10^{6}\right)$, transfected with the miR-182 or CXCR4 mimic, were injected subcutaneously into the hind-flank of six-week-old female nude mice ( $\mathrm{n}=5$ per group). Tumor formation and metastasis were followed for 35 days after the surgery using in vivo bioluminescence imaging (IVIS Imaging System PerkinElmer, Waltham, USA). The same number $\left(10^{6}\right)$ of HepG2 cells were injected into the right lower lobe of the livers in mice $(n=5)$ to assess metastasis. Animals were sacrificed on day 56, and tumor nodules counted in the left lobe.

\section{Results}

The TargetScan and microCosm algorithms were used to find miRNAs that might target MTA1. MiR-183 was one of the predicted miRNAs (Fig. 1A). Importantly, the miR-183 target site within the MTA1 3'UTR was conserved across species (Fig. 1B).

To estimate the expression of MTA1 in tumor specimens with high and low miR-183 expression levels, we surveyed 10 pairs of tumor and adjacent normal tissue samples from breast, renal, prostate, and testicular cancers for miR-183 expression. Following identification of low and high expressing specimens, we performed immunohistochemistry on representative samples. Significantly higher MTA1 expression was detected in tumor specimens with low miR-183 levels than in those with high miR-183 expression, suggesting 


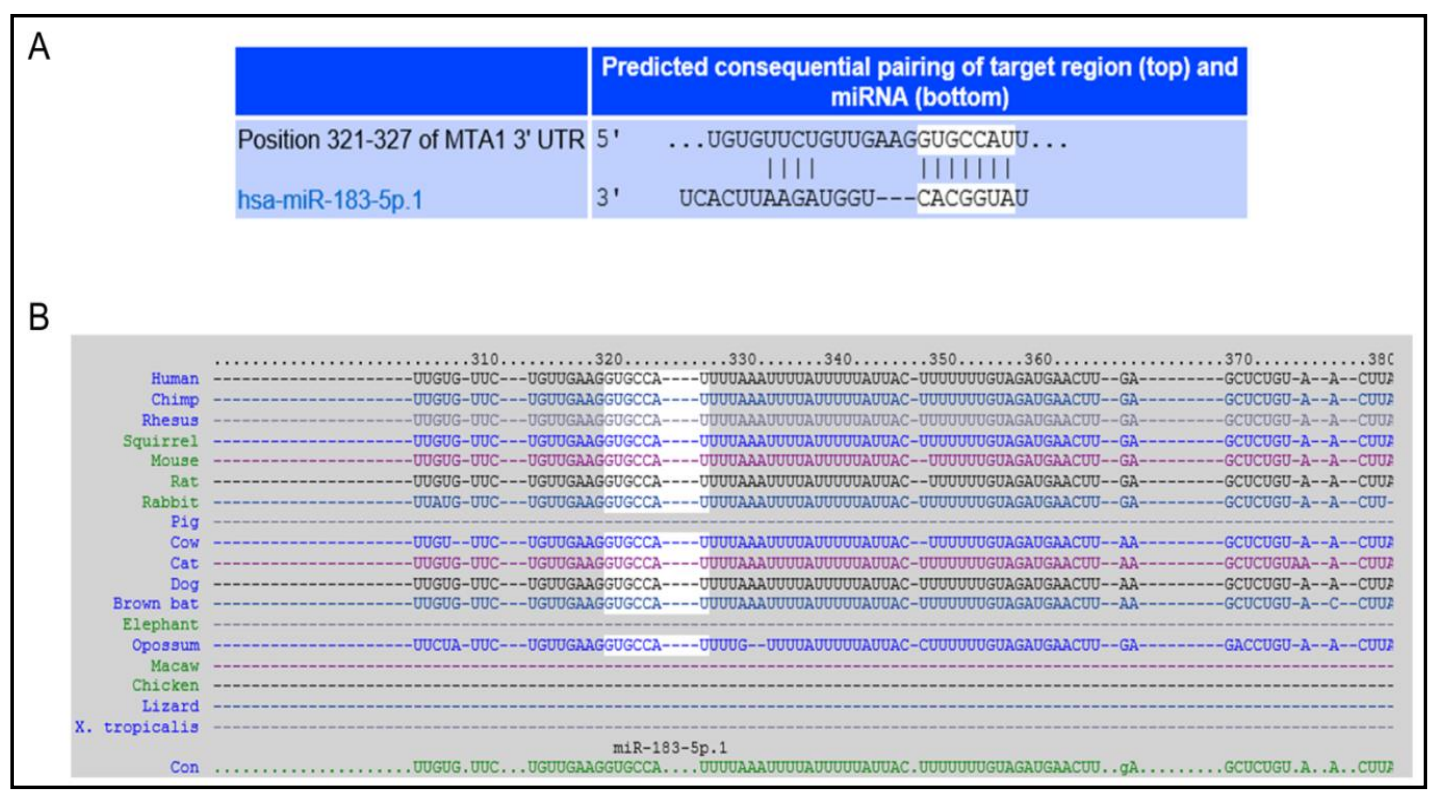

Fig. 1. Prediction of MTA1 as a target of miR-183-5p.1. (A) Complementary 7mer-m8 seed match between miR-183 and the $3^{\prime}$ UTR of MTA1 as predicted by TargetScan software. (B) Conservation of the 7 mer-m8 seed of miR-183 in the 3'UTR of MTA1 in indicated organisms.

Fig. 2. MTA1 and miR-183 expression are inversely correlated in patients with different cancer types. Representative im munohis to chemistry images of MTA1 expression in breast $(A, B)$, renal $(C, D)$, prostate $(\mathrm{E}, \mathrm{F})$, and testicular $(\mathrm{G}, \mathrm{H})$ cancer tissue samples with low and high expression

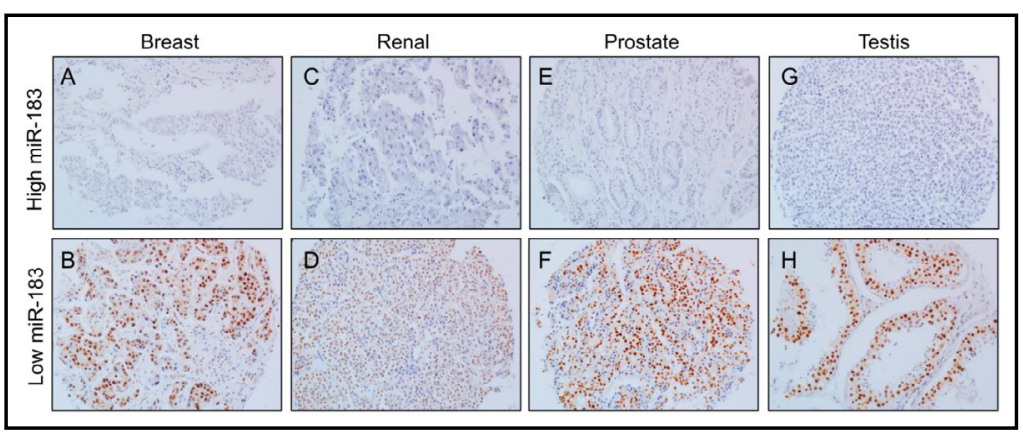
of miR-183. Images were obtained at 10X magnification.

Fig. 3. MTA1 is a bona-fide target of miR-183 in the HCC cell line HepG2. (A) Steady state expression of miR-183 and MTA1 in HepG2 cell line was determined. Data was normalized to RNU6B and TBP expression, respectively. (B)

Relative luciferase activity of transiently transfected luciferase reporter constructs containing either fulllength or mutated (miR-183 binding site deleted) MTA1 3' UTR in HepG2 cells, either mock transfected or transfected with miR-183 mimic.

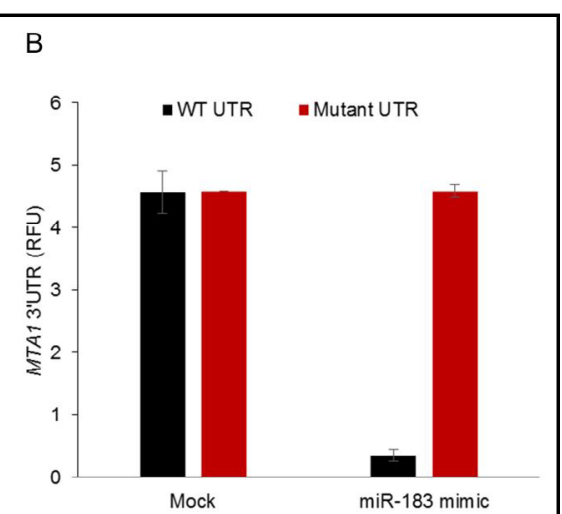


that MTA1 may be a putative target of miR-183 (Fig. 2), suggesting that MTA1 might be a putative target of miR-183.

We then set out to prove that MTA1 is indeed a target of miR-183. We evaluated HCC cell lines for miR-183 and MTA1 expression. One of the cell lines that had a suppressed miR-183 expression (-12.8 \pm 0.3 fold) and a correspondingly high MTA1 expression ( $+3.1 \pm 0.4$ fold) was HepG2 (Fig. 3A). To confirm the interaction between miR-183 and the 3'UTR of MTA1, we transfected renilla reporter plasmids harboring the MTA1 3'UTR into HepG2 cells, either alone or with the miR-183 mimic (Fig. 3B). The miR-183 mimic suppressed reporter activity of the MTA1 3'UTR by $4.6 \pm 0.05$ fold (P = 0.004) in HepG2 cells. In contrast, upon testing a miR-183 binding mutant of the MTA1 3'UTR reporter (in which nucleotides 321-327 - the miR-183 binding site - were deleted), we found that the miR-183 mimic could no longer repress the mutant reporter activity ( $p>0.05$ ) (Fig. 3B), confirming MTA1 as a direct target of miR-183.

Transfection with miR-183 mimic did not suppress cell proliferation after day 1 (CXCR vs miR-183 mimic $-0.67 \pm 0.04$ vs $0.68 \pm 0.05$, $p>0.05$ ), day 2 (CXCR vs miR-182 mimic -1.13 \pm 0.04 vs $0.43 \pm 1.13, \mathrm{p}>0.05$ ), and day 3 (CXCR vs miR-182 mimic $-2.04 \pm 0.06$ vs $2.04 \pm$ 0.09 , $\mathrm{p}>0.05$ ) (Fig. $4 \mathrm{~A}$ ).

HepG2 cells transfected with CXCR4 or miR-183 mimic were used to measure in vitro migration (Fig. 4B) and invasion (Fig. 4C) assays. Overexpression of miR-183 inhibited in vitro migration (CXCR vs miR-183 mimic $-75 \pm 3$ vs $25 \pm 2$, p $<0.05$ ), and invasion (CXCR vs miR-183 mimic $-73 \pm 5$ vs $21 \pm 4, p<0.05$ ). The results indicated that decreased levels of miR-183 would lead to increased in vitro migration and invasion in HCC cells, through the upregulation of MTA1. Taken together, the decrease in the relative expression of miR-183 and the corresponding increase in MTA1 expression in HCC tissues or cell lines, along with its capacity to regulate in vitro migration and invasion implied that miR-183 could potentially inhibit tumorigenesis and metastatic progression in HCC [14].

We used HepG2 cells stably overexpressing CXCR4 mimic or miR-183 mimic in a subcutaneous xenograft assay to assess the in vivo consequences of high miR-183 expression on tumor growth. Our results showed that ectopic overexpression of miR-183 in the HepG2 cells significantly inhibited tumor growth rate (Fig. 5A) $(\mathrm{P}<0.01)$ and decreased tumor burden (data not shown), compared to the HepG2/CXCR4 mimic xenografts. Using a hepatic tumor growth model, we then determined whether miR-183 also regulated metastasis in HCC. We found that the stable overexpression of miR-183 in HepG2 cells downregulated formation of hepatic tumor nodules in the non-injected liver lobes (Fig. 5B). The present results indicating that miR-183 functions as a tumor suppressor in HCC is consistent with its action of downregulating MTA1, which is both pro-tumorigenic and pro-metastatic in HCC.

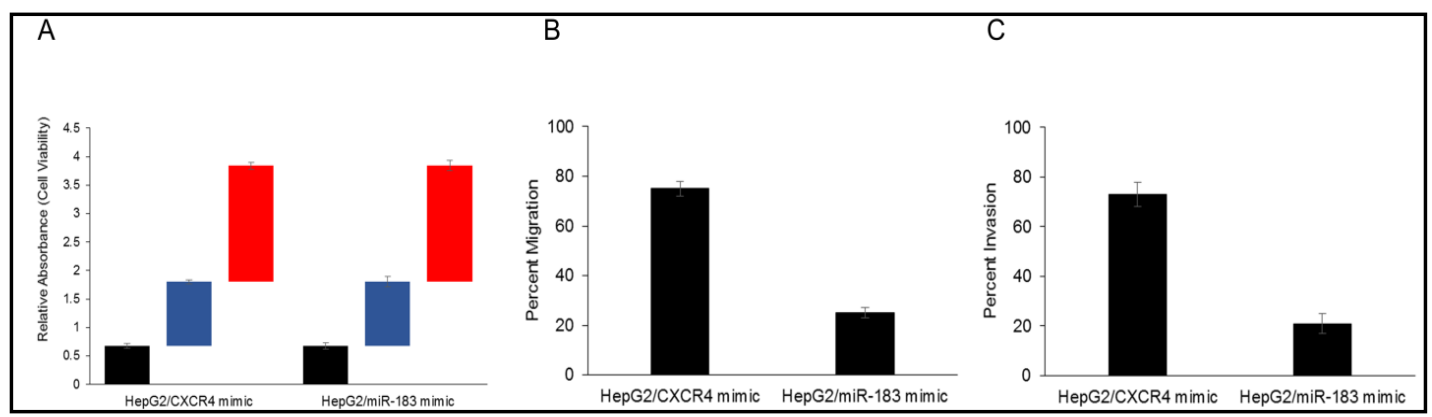

Fig. 4. MiR-183 expression levels dictate in vitro migration and invasion abilities in HCC cell lines without affecting cell proliferation. (A) Cell viability was measured in HepG2 cells transfected with CXCR4 mimic or miR-183 mimic at 24, 48, and 72 hours after transfection by the MTT assay. (B, C) Modulation of miR183 changes cell migration and invasion abilities in the HepG2 cells. The migrated and invasive cells were photographed using a microscope, and the number of the migrated (B) and invasive (C) cells in every field was counted and represented as percent of total cells at the beginning of the assay. Error bars, S.D. 
Finally, we performed metagenomic analysis of The Cancer Genome Atlas (TCGA) data and identified an inverse correlation between MTA1 expression and miR-183 expression (Fig. 6A). In fact, when we determined miR-183 and MTA1 expression in 25 HCC patients, the results indicated an inverse relationship between the levels of miR-183 and that of MTA1 in HCC tissue specimens (Fig. 6B) $(\mathrm{P}<.005$, Pearson correlation $r=-0.8318)$.

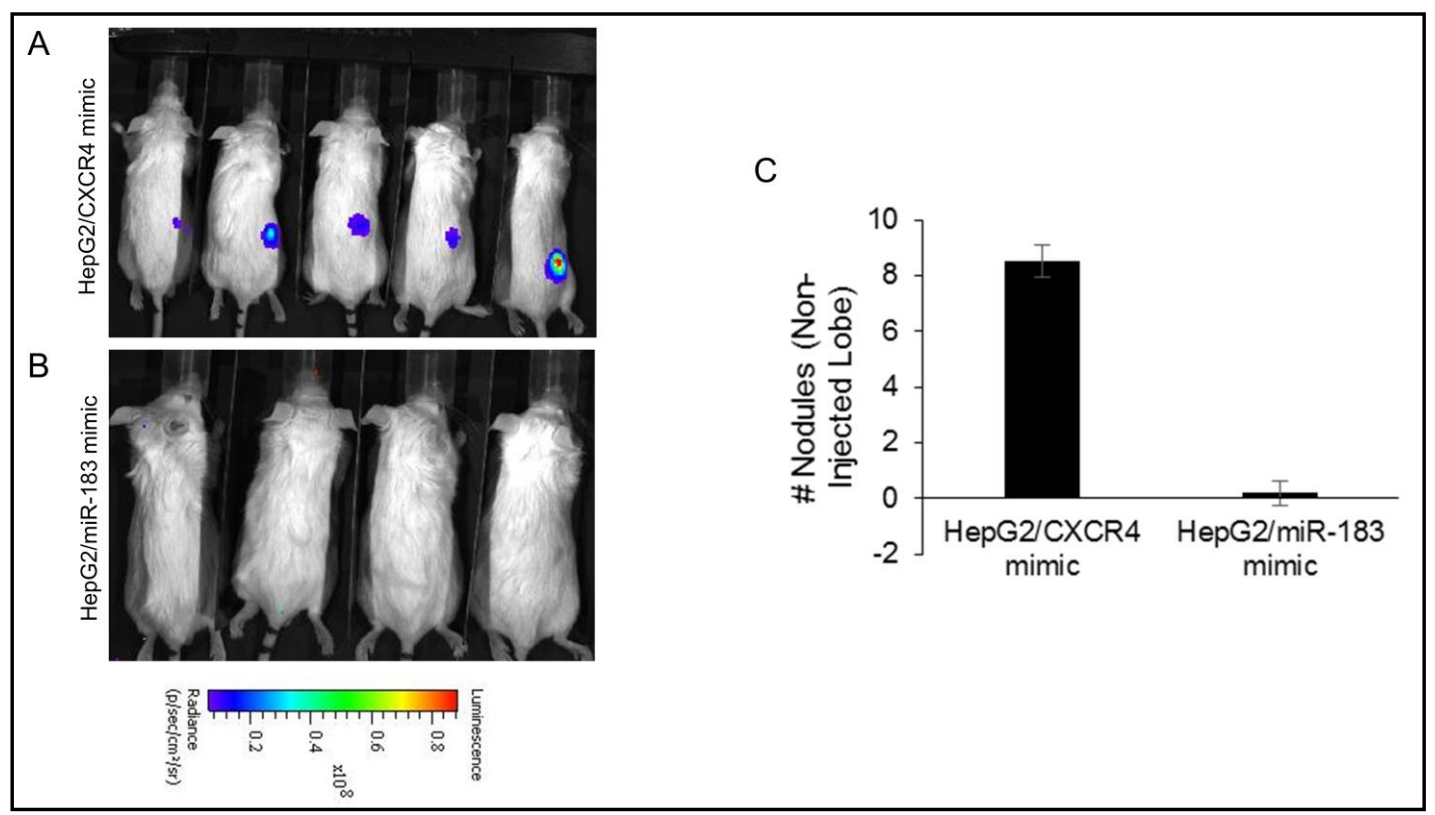

Fig. 5. MiR-183 expression levels correlate with metastatic potential of HCC cell lines in vivo. (A) Firefly luciferase expressing HepG2 cells, stably transfected with either CXCR4 mimic or miR-183 mimic were intravenously injected subcutaneously in $\mathrm{p} 53^{\%}$ mice. Mice were imaged by in vivo luciferase imaging up to 8 weeks to detect tumor formation. Tumor formation were observed in all animals injected with the HepG2/ CXCR4 mimic injected cells and was significantly attenuated in animals injected with the HepG2/miR-183 mimic. (B) The effects of miR-183 on hepatic burden were investigated. Animals injected with HepG2/miR183 mimic cells had a markedly attenuated hepatic tumor burden compared to HepG2/CXCR4 mimic as determined by measuring the occurrence of hepatic nodules in liver lobes other than the injected lobe.

Fig. 6. Expression of miR-183 and MTA1 is inversely correlated in hepatocellular cancer. (A) Metagenomic analysis of expression of miR-183 and MTA1 in HCC patients. Heat map depicts $\log _{2}$-fold change in mRNA and miRNA expression of indicated genes between tumornormal matched pairs for 95 TCGA patients. (B) MTA1 mRNA and miR-183 expression are inversely correlated in patients with HCC. Pearson correlation demonstrating the inverse relation between MTA1 and miR-183 in paired samples $(\mathrm{P}<0.05$, Pearson correlation $r=-0.8318)$.

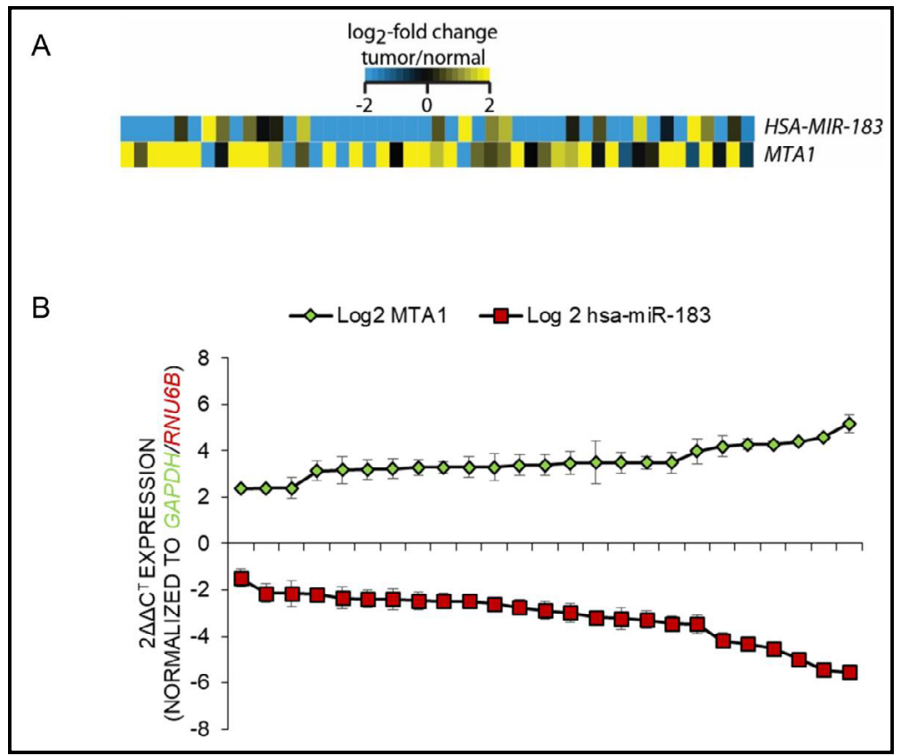




\section{Discussion}

The present results showed that miRNA-183 and MTA1 expression are inversely regulated in multiple human cancers including HCC and indicate that miRNA-183 acts by downregulating MTA1. Increasing the levels of miRNA-183 through transfection by a mimic did not affect cell proliferation but suppressed in vitro migration and invasion, as well as well as in vivo tumorigenesis and metastatic potential in the HepG2 HCC cell line. Thus, the present results establish miR-183 as a tumor suppressor in HCC. Given that the current study focused on patients without any prior surgical or therapeutic intervention, it will be important to examine and understand how such interventions affect the balance between miR-183 and MTA1 expression in patients with HCC. Furthermore, it will be clinically relevant to investigate if replenishment of miR-183 to levels observed in normal hepatocytes would increase the susceptibility of HCC cells to chemotherapy.

The unique aspect of miRNAs is that they can function both as tumor suppressors as well as oncomirs in a context-dependent fashion $[15,16]$. Similarly, miRNAs have been shown to work both as facilitators and inhibitors of tumor metastasis [17-22].

Our findings directly contradict previous reports of high miR-183 expression in HCC [23-25]. In one study, it was shown that over-expression of miR-183/96/182 confers prooncogenic properties to HCC [24]. Another study showed that miR-183 is overexpressed in HCC, but not associated with changes in patient survival [23]. Whether the discrepancies among the results can be explained by miR-183 acting as tumor suppressor under some conditions and as pro-oncogene under others, awaits further study.

\section{Abbreviations}

MTA1 (Metastasis associated 1); HCC (hepatocellular carcinoma); 3' (UTR,3'untranslated region); MTAs (Metastasis-associated genes); EMT (epithelial-mesenchymal transition); miRNAs (MicroRNAs); qRT-PCR (quantitative real time polymerase chain reaction); PCR (polymerase chain reaction); SD (standard deviation); OD (optical density).

\section{Acknowledgements}

This study was supported by the Medical Applicable Technology Tracking Project from Department of Health of Hebei Province (Precision Liver Resection, gl2010-15). W. Y. W. and W.B. designed the study; H. F. Z, M. T., S. J. Z, L. C. W. and L. L. L. conducted the experiments; M. T. performed data analysis; W. B. prepared the manuscript; and W. Y. W. performed statistical analysis. All authors read and approved the final manuscript.

\section{Disclosure Statement}

The authors declare that they have no conflict of interests.

\section{References}

1 Siegel R, Naishadham D, Jemal A: Cancer statistics, 2013. CA Cancer J Clin 2013;63:11-30.

- Hanahan D, Weinberg RA: Hallmarks of cancer: the next generation. Cell 2011;144:646-674.

-3 Farazi PA, DePinho RA: Hepatocellular carcinoma pathogenesis: from genes to environment. Nat Rev Cancer 2006;6:674-687.

-4 Aravalli RN, Steer CJ, Cressman EN: Molecular mechanisms of hepatocellular carcinoma. Hepatology 2008;48:2047-2063. 


\section{Cellular Physiology Cell Physiol Biochem 2018;51:2065-2072

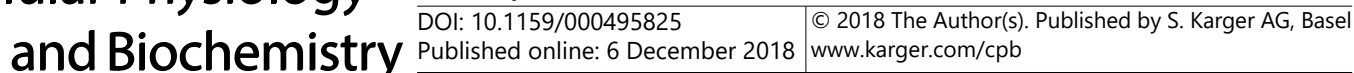

5 Tang ZY, Ye SL, Liu YK, Qin LX, Sun HC, Ye QH, Wang L, Zhou J, Qiu SJ, Li Y, Ji XN, Liu H, Xia JL, Wu ZQ, Fan J, Ma ZC, Zhou XD, Lin ZY, Liu KD: A decade's studies on metastasis of hepatocellular carcinoma. J Cancer Res Clin Oncol 2004;130:187-196.

6 Toh Y, Pencil SD, Nicolson GL: A novel candidate metastasis-associated gene, mta1, differentially expressed in highly metastatic mammary adenocarcinoma cell lines. cDNA cloning, expression, and protein analyses. J Biol Chem 1994;269:22958-22963.

7 Toh Y, Nicolson GL: The role of the MTA family and their encoded proteins in human cancers: molecular functions and clinical implications. Clin Exp Metastasis 2009;26:215-227.

$>8$ Bowen NJ, Fujita N, Kajita M, Wade PA: Mi-2/NuRD: multiple complexes for many purposes. Biochim Biophys Acta 2004;1677:52-57.

-9 Nicolson GL, Nawa A, Toh Y, Taniguchi S, Nishimori K, Moustafa A: Tumor metastasis-associated human MTA1 gene and its MTA1 protein product: role in epithelial cancer cell invasion, proliferation and nuclear regulation. Clin Exp Metastasis 2003;20:19-24.

10 Ryu SH, Chung YH, Lee H, Kim JA, Shin HD, Min HJ, Seo DD, Jang MK, Yu E, Kim KW: Metastatic tumor antigen 1 is closely associated with frequent postoperative recurrence and poor survival in patients with hepatocellular carcinoma. Hepatology 2008;47:929-936.

11 Deng L, Yang H, Tang J, Lin Z, Yin A, Gao Y, Wang X, Jiang R, Sun B: Inhibition of MTA1 by ERalpha contributes to protection hepatocellular carcinoma from tumor proliferation and metastasis. J Exp Clin Cancer Res 2015;34:128.

12 Hu S, Wilson KD, Ghosh Z, Han L, Wang Y, Lan F, Ransohoff KJ, Burridge P, Wu JC: MicroRNA-302 increases reprogramming efficiency via repression of NR2F2. Stem Cells 2013;31:259-268.

13 Card DA, Hebbar PB, Li L, Trotter KW, Komatsu Y, Mishina Y, Archer TK: Oct4/Sox2-Regulated miR-302 Targets Cyclin D1 in Human Embryonic Stem Cells. Mole \& Cell Biol 2008;28:6426-6438.

$>14$ Huang Z, Cheng L, Guryanova OA, Wu Q Bao S: Cancer stem cells in glioblastoma--molecular signaling and therapeutic targeting. Protein Cell 2010;1:638-655.

15 Bartel DP: MicroRNAs: target recognition and regulatory functions. Cell 2009;136:215-233.

16 Esquela-Kerscher A, Slack FJ: Oncomirs - microRNAs with a role in cancer. Nat Rev Cancer 2006;6:259-269.

17 Aleckovic M, Kang Y: Regulation of cancer metastasis by cell-free miRNAs. Biochim Biophys Acta 2015;1855:24-42.

18 Gaur A, Jewell DA, Liang Y, Ridzon D, Moore JH, Chen C, Ambros VR, Israel MA: Characterization of microRNA expression levels and their biological correlates in human cancer cell lines. Cancer Res 2007;67:2456-2468.

19 Kumar MS, Lu J, Mercer KL, Golub TR, Jacks T: Impaired microRNA processing enhances cellular transformation and tumorigenesis. Nat Genet 2007;39:673-677.

-20 Lu J, Getz G, Miska EA, Alvarez-Saavedra E, Lamb J, Peck D, Sweet-Cordero A, Ebert BL, Mak RH, Ferrando AA: MicroRNA expression profiles classify human cancers. Nature 2005;435:834-838.

21 Yang CL, Zheng XL, Ye K, Ge H, Sun YN, Lu YF, Fan QX: MicroRNA-183 Acts as a Tumor Suppressor in Human Non-Small Cell Lung Cancer by Down-Regulating MTA1. Cell Physiol Biochem: 2018;46:93.

-22 Xie X, Ma L, Xi K, Zhang W, Fan D: MicroRNA-183 Suppresses Neuropathic Pain and Expression of AMPA Receptors by Targeting mTOR/VEGF Signaling Pathway. Cell Physiol Biochem 2017;41:181.

23 Li ZB, Li ZZ, Li L, Chu HT, Jia M: MiR-21 and miR-183 can simultaneously target SOCS6 and modulate growth and invasion of hepatocellular carcinoma (HCC) cells. Eur Rev Med Pharmacol Sci 2015;19:32083217.

24 Liang Z, Gao Y, Shi W, Zhai D, Li S, Jing L, Guo H, Liu T, Wang Y, Du Z: Expression and significance of microRNA-183 in hepatocellular carcinoma. Sci World J 2013;2013:381874.

-25 Leung WKC, He M, Chan AWH, Law PTY, Wong N: Wnt/ $\beta$-Catenin activates MiR-183/96/182 expression in hepatocellular carcinoma that promotes cell invasion. Cancer Lett 2015;362:97-105. 